\title{
Erratum: Chest radiographs - Lines, tubes, non-cardiac medical devices and materials
}

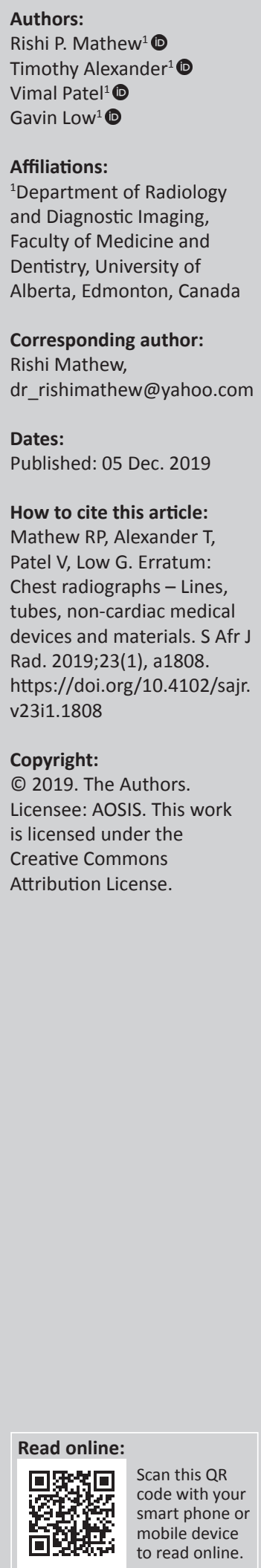

In the initial version of this article published earlier, the article title was incorrect. The title is hereby updated as 'Chest radiographs - Lines, tubes, non-cardiac medical devices and materials'.

This correction does not alter the study's findings of significance or the overall interpretation of the study results. The publisher apologises for any inconvenience caused. 